\title{
Efficient End-to-End Transport of Soft Real-Time Applications ${ }^{\star}$
}

\author{
Zoe Antoniou ${ }^{1}$ and Ioannis Stavrakakis ${ }^{2}$ \\ 1 Nokia Research Center \\ 3 Burlington Woods Drive, Burlington, MA, 01803, USA \\ zoe.antoniou@nokia.com \\ 2 Department of Informatics, University of Athens \\ Athens, 15784, Greece \\ istavrak@di.uoa.gr
}

\begin{abstract}
Typically, real-time applications are well supported in environments where there is ample bandwidth available, enabling their transport within the stringent time constraints. Accordingly, non-realtime applications are well supported in environments where there is ample storage available resulting in low losses. For an emerging class of applications such as prerecorded video - referred to as soft real-time applications there may be an increased role that storage can play - in addition to bandwidth - in their effective transport. A scheme is proposed in this paper that takes advantage of available storage within the network and the end users, to efficiently transport prerecorded continuous media traffic across a number of network nodes.

The scheme is based on a scheduling policy implemented within the network nodes called Deadline Credit (DC) policy. Its objectives are (a) to utilise all (if possible) of the available capacity by allocating bandwidth dynamically to a number of data streams, (b) to distribute the available bandwidth fairly between all competing data streams and (c) to maximise the number of Application Data Units (ADUs) that are served by always giving priority to the one with the shortest time to expiration. Numerical results are presented and the induced ADUs loss rates under the proposed policy are compared against those of some alternative schemes.
\end{abstract}

\section{Introduction}

The transmission of continuous media traffic is an inherently difficult problem due to the time sensitive nature and the traffic variability of these applications [1], 2].

Consider, for instance, the transmission of video applications in which it is required that frames (images) be transmitted at a certain rate in order to

\footnotetext{
* This work is part of Zoe Antoniou's Ph.D. research while with Northeastern University and has been supported by the National Science Foundation under Grant NCR 9628116.
} 
guarantee no starvation or overflow at the receiving end. Bits generated over a frame form a logical block of information (Application Data Unit or ADU) which is central to both the traffic generation process at the transmitter and the reconstruction process at the receiver. Such a role may be played by a smaller encoding unit than the frame such as a macroblock [3. Since frames are typically generated at a constant frame rate the nature of such an application is periodic.

ADUs can be mapped into a collection of network date units or ATM cells. In order to guarantee a timely reconstruction at the receiver, complete frames should be delivered at a constant display rate. This implies that all cells of an ADU should be delivered in time for the reconstruction process in order to avoid either starvation or buffer overflow at the receiving end. When network resources are reserved at peak demand, all cells of the ADU will be delivered on time, leading to a timely and error-free reconstruction at This is clearly the case when resources are over-allocated to a number of applications to increase network utilisation.

The requirement for timely delivery of information associated with such continuous media traffic suggests that deadlines could be defined and deadlinedriven policies be designed. If the nature of an application requires that a complete ADU should be received before it can be processed by the receiver, then it would be reasonable to associate a common deadline with all the cells in the same data unit and develop scheduling policies that aim to maximise the number of complete data units that are delivered by their respective due date. This approach is different from many traditional approaches in which every cell is assigned its own deadline and the scheduling policy typically attempts to maximise the number of individual cells transmitted by their due date [4], 5].

Continuous media traffic may be real time or prerecorded such as Video on Demand (VoD). In the prerecorded case, which is the focus of this paper, there can be various alternatives to the efficient transmission of the data since the information is available a priori, as opposed to the real time traffic where real time processing and transmission is the only approach. In either case, they both have a periodic consumption rate once the application starts running.

The proposed policy, called Deadline Credit (DC) policy, focuses on the efficient scheduling of soft real-time constrained applications. Its first objective is to utilise all (if possible) of the available capacity by allocating bandwidth dynamically to a number of (continuous media) data streams. Secondly, it aims to distribute the available bandwidth fairly between all competing data streams. Finally, it aims to maximise the number of ADUs that are served by always giving priority to the one with the shortest time to expiration. In order to achieve this the DC policy uses all of the available bandwidth to (a) serve the ADUs with upcoming deadlines and (b) send ADUs in advance in order to build deadline credit. This deadline credit can be consumed later when the demand for resources may increase.

In this paper, the DC approach is described for a multihop network for the case of prerecorded traffic. It is an extension of the DC policy described in [6]. For time critical applications, such as continuous media applications, the most 
important resource is high bandwidth availability in order to achieve timely delivery of information. Traditionally, large buffer space has not been very useful in this environment. Bandwidth becomes an even more scarce resource when the demand increases and the system is utilised near its full capacity.

In the case of the DC approach, however, large buffers can help improve the performance of soft real- time constrained applications. When the demand for bandwidth is low ADUs can be sent in advance to the downstream nodes in the process of building up the deadline credit of the stream (assuming buffer availability). Naturally, the larger the available buffer space the higher is the potential for the deadline credit build up. In turn, the higher the deadline credit build up the higher the number of ADUs whi ch are "forwarded" in advance towards the destination. Also, the more links the ADUs traverse in advance the closer to the destination they reach.

Consequently, large buffers can be useful in forwarding as many ADUs as possible, as close to the destination as possible in advance in an attempt to exploit the available bandwidth when the demand is low. This way, congestion is expected to be alleviated or avoided at a later time when the demand may increase. This is the driving motivation behind applying the DC approach to a multihop network.

In [7] an overview of some related policies is presented. Among others, the problem of scheduling and transmitting prerecorded traffic and, in particular, video has been addressed in [3], [?] where a policy called Constant-Rate Transmission and Transport (CRTT) was proposed. According to CRTT, a number of frames (ADUs) are sent in advance (prior to the commencement of playback). This provides an initial "build up" and enables the remaining frames to be transmitted at a constant rate during playback. The amount of "build up" and the rate of transmission are computed given the characteristics of the movie which are known a priori and the amount of buffering available at the receiving end. However, the issue of multiplexing a number of streams carrying prerecorded traffic or how the losses will be distributed between streams sharing the same link for high network utilisation is not directly addressed as it is for the policy proposed here. In addition, the allocation of network resources is static and is determined once at the beginning of the session, as opposed to the dynamic allocation performed by the DC policy based on the varying network resource requirements from all users. The CRTT policy is rate based and deadlines are not explicitly defined as opposed to the DC policy.

\section{The DC Policy}

In this paper a multihop network of source, intermediate and destination nodes (with bidirectional links) is considered. A node can have a number of incoming and outgoing links. Each link arrives at (or leaves from) a port. Ports for incoming links have an amount of buffer space allocated to the streams that arrive at this port. Ports for outgoing links have a scheduler/server. Streams are multiplexed and transmitted by the scheduler/server onto the correct output link 
in order to reach to the correspondin g receiver where they are decoded and reconstructed. Source nodes also have a mass storage device and a file server. The issues associated with retrieving streams of data from a storage device are beyond the scope of this paper.

The system is assumed to be slotted; a cell requires a fixed amount of time for transmission referred to as a slot. All departures from the node occur at slot boundaries. Let $j$ denote a data stream (application); $j$ takes values in the range $0 \leq j \leq J-1$. A variable with superscript $j$ represents a quantity associated with stream $j$. Let $n$ denote a node; $n$ takes values in the range $1 \leq n \leq N$. A variable with subscript $n$ represents a quantity associated with node $n$. Also, let $P_{i}^{j}$ denote the $i^{\text {th }}$ ADU from stream $j$ which corresponds to the $i^{\text {th }}$ periodic interval of the session of stream $j$; let $\hat{P}_{i}^{j}$ represent its length in cells. (Subscript $n$ is not used with variable $P$.)

The per stream (application) traffic is assumed to be bursty in nature. It consists of application data units, ADUs; each ADU is of variable length (measured in cells). For real time traffic a new ADU is assumed to be generated periodically every $T^{j}$ slots, where $T^{j}$ denotes the period of the generation process of the application measured in slots. The $i^{\text {th }}$ ADU is generated at the $i^{\text {th }}$ periodic interval. So for real time traffic the number of ADUs available to the server at any time is limit ed by the generation process rate of the application. For prerecorded traffic though, all of the ADUs of the session are available at the source node prior to the commencement of transmission.

The application is assumed to have a periodic reconstruction rate; an ADU has to be available to the receiver every $T^{j}$ slots for processing $\left(T^{j}\right.$ is assumed to be both the generation and the reconstruction periodic interval of the application). So each application has a maximum allowable end-to-end delay, $E T E^{j}$, which is defined to be the same for all ADUs of stream (application) $j$. This $E T E^{j}$ delay is the sum of all propagation delays between nodes, and the sum of all queuing and trans mission delays (called node delays in this paper) at each node for stream $j$. Propagation delays depend on link capacities, physical distance between nodes and the number of hops. If the sum of all propagation delays is subtracted from $E T E^{j}$, the remaining is the total amount of time an ADU can spend on queuing and transmission delays in all the nodes. This amount of time can be distributed in a variety of ways between nodes. For instance, it can be distributed equally between nodes or based on their utilisation. The distribution can be static (once upon connection set-up) or dynamic (varying based on the load of each node during the session). As it will be clarified in section 2.1, in this paper the node delays are defined cumulatively from one node to the next. If part of the node delay is not used in one node it is available to be used at a later node.

The QoS metric considered here is the ADU loss probability. An ADU is assumed to be lost if any one of its cells is lost. The DC policy is work conserving in the sense that it will serve as long as there are ADUs available for service and buffer space is available at the next downstream node. 
The proposed scheduling algorithm determines when the next stream is to be selected for service, which stream is to be selected and whether an ADU from the selected stream is to be served or dropped. The scheduling algorithm is executed at every outgoing port of each node. A set of metrics is used in making the above decisions, namely, the deadline credit, the losses and the priority index of the stream. A counter is associated with each metric per node $n$ an d stream $j$ : (a) the deadline credit counter, $D_{-} C R_{n}^{j}$, (b) the losses counter, $L_{-} C R_{n}^{j}$ and (c) the priority index counter, $P_{-} C R_{n}^{j}$. In addition to the three metrics two more parameters are used in making scheduling decisions: (d) the sequence number, $S N$ and (e) the buffer availability.

A sequence number is defined for each ADU of stream $j$. The definitions of the counters and parameters and their descriptions are given in the following subsections, which are followed by a description of the scheduling procedure. A more detailed description can be found in [7].

It is assumed that key events occur and actions are taken instantaneously at the end of slots. If during a slot either the last cell of an ADU or no cell is being served, the server will be idle at the end of this slot and this is referred to as a decision slot. At decision slots certain actions are taken leading to the selection of a stream to be served next. For the selected stream this decision slot becomes an examination slot; ADUs of stream $j$ can be scheduled for service or dropped only a t examination slots of stream $j$. The losses counter of a stream is updated only at the examination slots of this stream as it will be discussed later.

The ADU at the head of stream $j$ is defined to be the ADU of stream $j$ which has the earliest due date among all ADUs of stream $j$ not served or dropped yet. As it will become clear later, expired ADUs can either remain in the system until the next examination slot for the corresponding stream at which time they are dropped or they can be dropped upon expiration and all the relevant counters can be updated.

\subsection{The Deadline Definition}

An end-to-end delay, $E T E^{j}$, is defined for each stream $j$. This consists of the total propagation delay from source to destination (from node 1 to node 2 , PROP_DELAY $Y_{1,2}^{j}$, from node 2 to node 3, PROP_DELAY $Y_{2,3}^{j}$, and so on), plus the maximum allowed queuing and transmission delay at each node (for node 1 NODE_DELAY $Y_{1}^{j}$, for node $2 N O D E_{-} D E L A Y_{2}^{j}$ and so on). The sum of all propagation, queuing and transmission delays is equal to the end-to-end delay. So ADU $P_{i}^{j}$ i s needed at the receiver $E T E^{j}$ slots after the instant marking the beginning of the $i^{t h}$ periodic interval of stream $j$ or $\left(i \cdot T^{j}+E T E^{j}\right)$ slots after the beginning of the session of stream $j$.

Using the assigned node and propagation delay tolerances a deadline is defined per stream and node. The deadline is defined cumulatively from one node to the next such that if an ADU leaves a node before the expiration of its deadline by an amount of $t$ slots, these slots are automatically available at later nodes in addition to their default deadline/delay tolerance where there they may be 
used if there is higher contention for bandwidth. If an ADU can not meet its deadline at any node it is dropped.

The deadline of $P_{i}^{j}$ is defined as the number of time slots from the instant marking the beginning of the $i^{\text {th }}$ periodic interval of stream $j$ as follows:

- At node 1: $D_{1}^{j}=$ NODE_DELAY

- At node 2: $D_{2}^{j}=N O D E \_D E L A Y_{1}^{j}+P R O P \_D E L A Y_{1,2}^{j}+N O D E \_D E L A Y_{2}^{j}$ $=D_{1}^{j}+$ PROP_DELAY $Y_{1,2}^{j}+N O D E \_D E L A Y_{2}^{j}$

- At node 3: $D_{3}^{j}=D_{2}^{j}+$ PROP_DELAY ${ }_{2,3}^{j}+N O D E_{-} D E L A Y_{3}^{j}$

- At node $n: D_{n}^{j}=D_{n-1}^{j}+$ PROP_DELAY $Y_{n-1, n}^{j}+N O D E \_D E L A Y_{n}^{j}$

Dropping an ADU at a node $n$ due to the node deadline violation, $D_{n}^{j}$, does not imply that the $E T E^{j}$ delay would have been violated as well. There is always the possibility that this ADU could have met the $E T E^{j}$ delay if it experienced delays at later nodes that were less than their respective deadlines. In that case, the ADU would have been dropped unnecessarily but this is a consequence of the distribution of the end-to-end deadline among the involved nodes.

he $E T E^{j}$.

The Additional Delay Tolerance (ADT) of an ADU of stream $j$ is defined to be equal to the amount of remaining time until the ADU expires reduced by its default deadline $D_{n}^{j}$. That is if ADT is equal to $d_{-} c r$ for an ADU of stream $j$, this ADU will expire after $d_{-} c r+D_{n}^{j}$ slots.

\subsection{The Sequence Number}

The SN marks the sequence of ADUs of a particular stream $j$. The SN is stored in the header of the ADU. It is used to identify which ADU corresponds to which periodic interval in order to determine whether the ADU can meet its deadline or has expired and needs to be dropped.

More specifically, if an ADU can not meet its deadline at any node $n$ it will be dropped and the next ADU will be scheduled for service. The scheduler can determine the number of expired ADUs at the waiting queue of the current node from the value of the deadline credit counter of the node. In the downstream nodes however, the value of their deadline credit counter is not enough to accurately determine the number of $\mathrm{ADU}$ that were dropped at upstream nodes (if any). It is in this case that the scheduler nee ds to read the SN of the incoming ADUs to determine how many ADUs have been dropped at upstream nodes (if any) and make the necessary adjustments to all the relevant counters. The SN could be used in all cases to identify the sequence of ADUs but the above paragraph explained the case where it would be necessary. Its use will be clarified further after reading sections 2.4 and 2.5.

\subsection{The Buffer}

Ports for incoming links have an amount of buffer space allocated to the incoming streams. Buffer space may be assigned separately to each stream or collectively 
a set of streams. As part of the scheduling process each node, e.g. $n$, needs to know the amount of available buffer space of the next downstream node, e.g. $n+1$.

In this paper, it is assumed that buffer space is assigned separately per stream at each incoming port. This guarantees that when the buffer condition (introduced in section 2.4) holds no ADUs will be dropped due to buffer overflow. For implementation purposes, the buffer availability can be checked in the following way. Each node $n$ knows the total amount of buffer space available per stream at the next downstream node, $n+1$, referred to as $B u f f e r_{n+1}^{j}$. In addition, each node keeps a record of the total amount of data transmitted per stream measured in cells or bytes or any other appropriate unit, data $a_{n}^{j}$. Finally, each node requires from each downstream node that it transmits data to the amount of data it has transmitted, again measured per stream, referred to as $d a t a_{n+1}^{j}$. The buffer availability per stream in the next downstream node can be determined as follows:

$$
\text { Available_buffer }{ }_{n+1}^{j}=B u f f e r_{n+1}^{j}-\left(\operatorname{data}_{n}^{j}-\operatorname{data}_{n+1}^{j}\right)
$$

The above formula will account for all the ADUs currently in the waiting queue of node $n+1$ as well as all the ADUs that are currently traveling towards node $n+1$.

If buffer space were assigned collectively for the set of streams arriving at a node $n+1$ from the same node $n$ then it would still be possible to guarantee no buffer overflow using the above method. If, however, the buffer pool at a node $n+1$ is shared between streams arriving from different nodes then guaranteeing no buffer overflow may not be possible or it may require additional feedback of information between nodes, thus, increasing the processing overhead.

The amount of buffer space available at the next node at any instant determines how many ADUs can be sent in advance. Feedback is necessary to update upstream nodes on the value of the $d a t a_{n}^{j}$ parameter. It is assumed that feedback is available periodically, based on which a stream can build a maximum possible deadline credit from the current value of $D_{-} C R_{n}^{j}$ to $D_{-} C R_{n}^{j} M A X$ till the next buffer availability update. It is assumed that the buffer of the next node $n+1$ can store a maximum of $b$ data units according to the latest buffer feedback update. This corresponds to a session duration of $b T^{j}$ slots. So the difference between the current value of $D_{-} C R_{n}^{j}$ and the current value of $D_{-} C R_{n-}^{j} M A X$ represents the remaining buffer space of the corresponding receiving buffer based on the latest feedback update. In the following sections, $D_{-} C R_{n-}^{j} M A X$ is used to define the buffer condition when making scheduling decisions.

\subsection{The Deadline Credit Counter}

The purpose of the deadline credit counter is to keep track of the ADT of the ADU at the head of the corresponding stream. At the same time, the deadline credit counter indicates how far ahead or behind schedule is the service of the ADUs of the corresponding stream still in the system. ADUs do not need to carry 
the value of the deadline credit counter as they propagate from one node to the next. A deadline credit counter is defined for each stream $j$ and node $n$ that stream $j$ traverses, denoted by $D_{-} C R_{n}^{j}$. The counter is initialised and updated such that upon arrival of a new ADU at a node $n$, the value of $D_{-} C R_{n}^{j}$ is the same as the value of $D_{-} C R_{n-1}^{j}$ when transmission of the ADU was completed at node $n-1$. This enables the scheduler at any node $n$ to determine how far ahead or behind schedule is the service of the ADUs of stream $j$ in all the nodes up to and including node $n$ and use the value of the counter in making scheduling decisions.

At the beginning of session $j$, deadline credit counters are initialised at all the nodes that stream $j$ will traverse. The counter of each node is initialised such that the propagation delays and maximum allowable node delays of upstream nodes are accounted for. For instance, suppose that stream $j$ traverses nodes 1 through $N$. The deadline credit counters at each node are initialised as follows:

- Node 1: $D_{-} C R_{1}^{j}=0$

- Node 2: D_CR $R_{2}^{j}=D_{-} C R_{1}^{j}+N O D E \_D E L A Y_{1}^{j}+P R O P \_D E L A Y_{1,2}^{j}$

- Node 3: $D_{-} C R_{3}^{j}=D_{-} C R_{2}^{j}+N O D E \_D E L A Y_{2}^{j}+P R O P \_D E L A Y_{2,3}^{j}$

- Node n: D_C $R_{n}^{j}=D_{-} C R_{n-1}^{j}+N O D E_{-} D E L A Y_{n-1}^{j}+P R O P \_D E L A Y_{n-1, n}^{j}$

This is consistent with the deadline definitions as shown in section 2.1 The deadline credit counters are initialised cumulatively from one node to the next such that if the first ADU leaves a node before the expiration of its deadline by an amount of $t$ slots, these slots are automatically available at later nodes in addition to their default deadlines.

During the session $D_{-} C R_{n}^{j}$ is updated as follows:

(a) During every elapsed slot: $D \_C R_{n}^{j}=D_{-} C R_{n}^{j}-1$. This step captures the fact that any slot that passes by reduces the ADT of the ADU at the head of the queue of stream $j$ by one time slot.

(b) At the end of an examination slot for stream $j: D \_C R_{n}^{j}=D_{-} C R_{n}^{j}+(k+m) T^{j}$ where $k$ is all the dropped ADUs (which are all expired) and the binary (0/1) variant $m$ is 1 only if :(i) $D_{-} C R_{n}^{j}+(k+m) T^{j} \leq D_{-} C R_{n-}^{j} M A X$ (buffer condition), and (ii) $D_{-} C R_{n}^{j}+(k+m) T^{j}+D_{n}^{j} \geq \overline{\hat{P}}_{i}^{j}$ (deadline condition), where $P_{i}^{j}$ is the non expired ADU at the head of stream $j$.

Step (b) "adjusts" the context of $D_{-} C R^{j}$ to describe the ADT of the ADU at the head of stream $j$ after removing the dropped/scheduled for service ADUs of stream $j$. Step (b-(i)) checks the buffer availability and step (b-(ii)) checks the deadline condition for the ADU at the head of stream $j$.

\subsection{The Losses Counter}

In addition to the $D_{-} C R_{n}^{j}$ counter, a second counter, $L_{-} C R_{n}^{j}$, is set up to count the number of ADUs that are dropped from stream $j$ up until the last examination slot for stream $j . L_{-} C R^{j}$ is updated at examination slots for stream $j$ only as follows:

$$
L_{-} C R_{n}^{j}=L_{-} C R_{n}^{j}+k
$$


where $k$ is equal to the number of ADUs of stream $j$ dropped in the current examination slot for stream $j$. The dropped ADUs may belong to two categories: either ADUs that expired while waiting at node $n$, or ADUs that were dropped at an earlier node because their deadline could not be met.

As it was mentioned earlier, in the first case the value of the deadline credit counter at node $n$ is sufficient to determine the number of expired ADUs. In the second case, the scheduler uses the SN. In both cases, the losses counter, $L_{-} C R_{n}^{j}$, is updated with the losses for all the nodes up to and including node $n$ because: (a) losses that occur at previous nodes can be detected through the SN, and (b) losses that occur at node $n$ can be detected through the $D_{-} C R_{n}^{j}$ or the SN.

\subsection{The Priority Index Counter}

In order to guarantee that the available bandwidth will be distributed fairly between all competing streams, the two metrics are combined into a single metric, called priority index, that aims to capture both the current deadline credit of the stream and its cumulative losses. The priority index counter $P_{-} C R^{j}$ is defined

$$
P \_C R_{n}^{j}=\frac{D_{-} C R_{n}^{j}-T^{j} \cdot L_{-} C R_{n}^{j}}{T^{j}}
$$

The priority index counter represents the relative priority of competing data streams. The smaller the value of the priority index counter of a stream the higher the priority of the stream with respect to the others. This will be the stream with the most losses, if any, and among streams with no losses or the same losses it will be the stream with the lowest deadline credit.

\subsection{The Scheduling Algorithm}

The proposed algorithm is summarised for any node $n$ in the following flowchart. A more detailed and insightful description can be found in [7].

\section{Numerical Results and Discussion}

The performance of the DC policy is investigated under two different scenarios which demonstrate the improvement in the induced ADU loss rate between the DC policy and an alternative approach which permits no deadline credit build up. The alternative approach schedules only one ADU per period subject to buffer availability. In the following graphs, the ratio of the induced ADU losses is plotted as a function of the number of multiplexed streams.

The average bandwidth required by all streams, $U_{\text {req }}$, is defined as the sum of the average cell rate generation of all streams. So for a total of $N$ data streams:

$$
U_{\text {req }}=\sum_{j=0}^{N-1} \frac{\hat{P}_{a v e}^{j}}{T^{j}}
$$




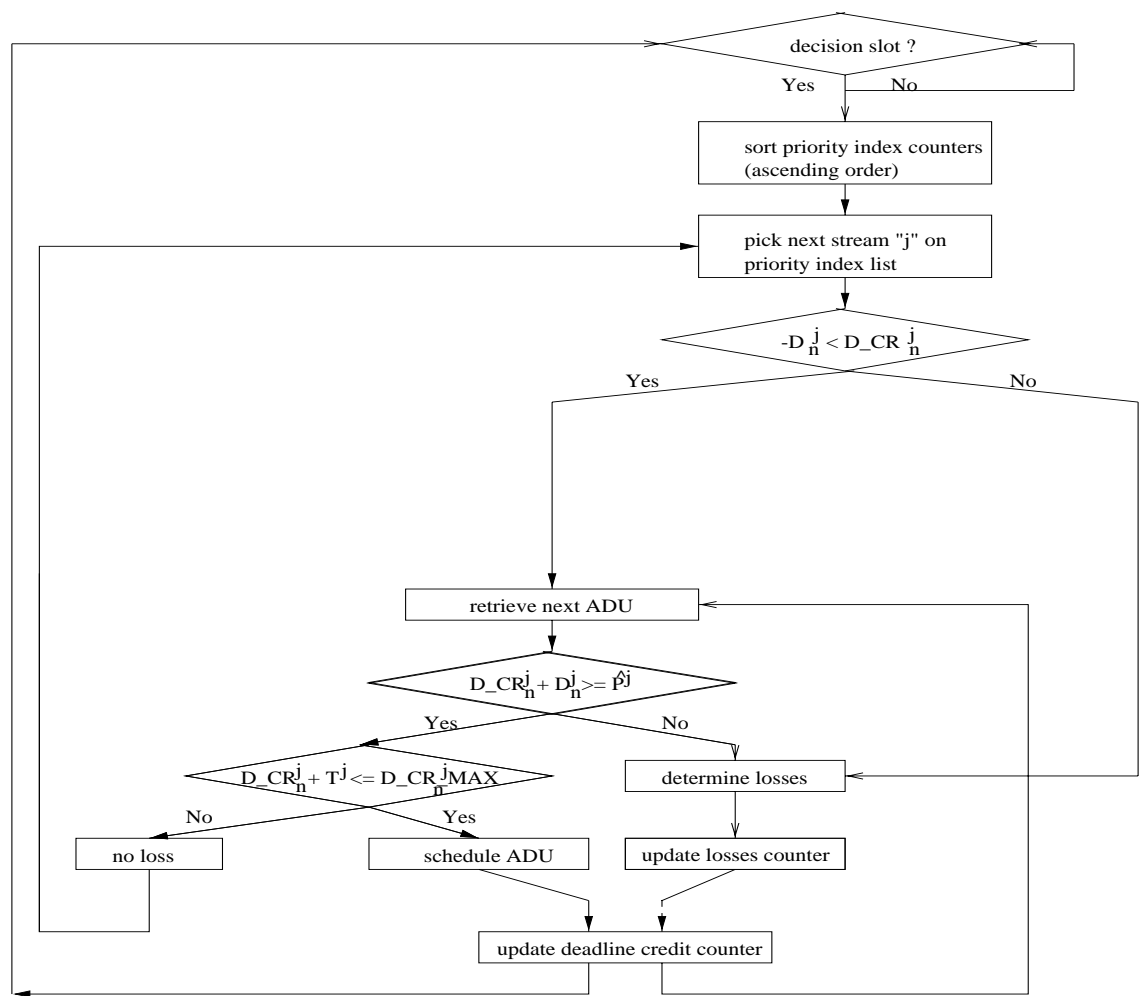

Fig. 1. The DC scheduling algorithm flowchart.

\subsection{Experiment 1}

The first scenario considers the case where a set of streams has the same source (node 1), destination (node 3 ) and the same intermediate nodes (node 2; only one intermediate node in this experiment). Its purpose is to show that the DC algorithm utilises the available bandwidth and buffer space and improves on the induced ADU losses as compared to the alternative policy. The intermediate and destination nodes are assumed to have the same amount of available buffer space per stream. The node delays are distr ibuted equally per node.

All streams, except from two background traffic streams, are assumed to have the same traffic characteristics. They have the same period, $T^{j}=100$ slots, the same ADU length distribution (uniform with maximum length $L P_{\max }=20$ cells), the same end-to-end deadline which is equal to one period, $E T E^{j}=T^{j}$ slots, and the same QoS requirements. The two background traffic streams have the same characteristics as above with the exception that the length of ADUs has a bursty distribution. It is equal to 1 cell with probability $p$ and equal to $L P_{\max }=20$ with probability $1-p$. For this experiment $p=0.3$, which implies that the background traffic streams generate long ADUs $70 \%$ of the time. 


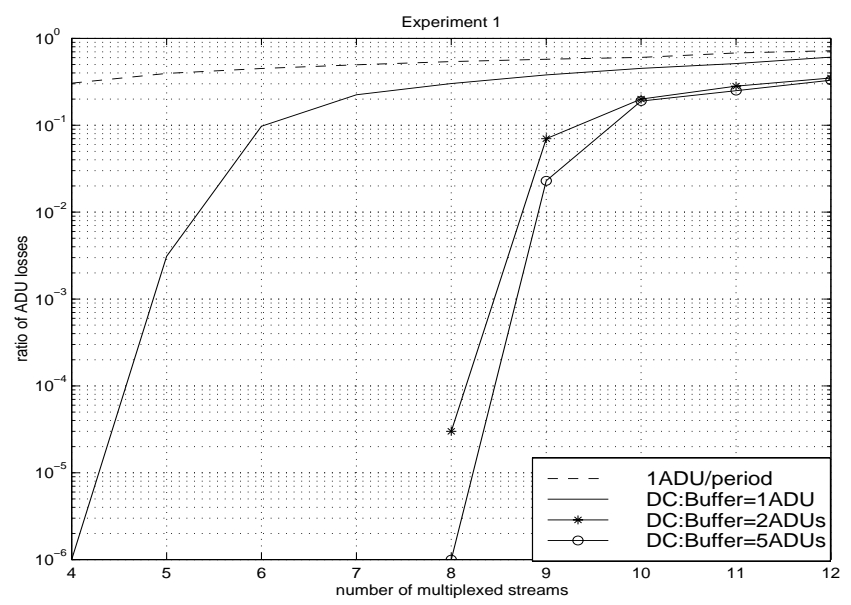

Fig. 2. Experiment 1: Ratio of induced ADU losses vs number of multiplexed streams.

In Fig. 2, the ratio of ADU losses under the DC policy is plotted for the cases where the available buffer space at each node is at most equal to $1 \mathrm{ADU}$, 2 ADUs and 5ADUs of maximum length per stream. Each stream requires an average of $10.5 \%$ of the link bandwidth; for example, 5 streams correspond to $52.5 \%$ of each link's utilisation.

For the DC policy, the case of Buffer $=1 A D U s$ is not very indicative of its performance as it allows for very limited deadline credit build up. With respect to the alternative approach of serving one ADU per period, there is a significant difference in the losses, especially for the cases of $B u f f e r=2 A D U s$ and Buffer $=5$ ADUs. For system utilisation approaching $100 \%$ the losses rates converge. This is expected since there is very little or no bandwidth available to be used for building deadline credit and, as a result, the available buffer space can not be utilised. So the losses are comparable in all cases.

\subsection{Experiment 2}

The second scenario considers the case where a set of streams with homogeneous traffic characteristics has to travel from the same source (node 1) to the same destination (node 3 ) through one intermediate node (node 2). In addition, there are background traffic streams (in this case three streams) which travel from node 1 to node 2 . At node 2, they can be assumed to terminate or continue on to another link, separately from the rest of the streams. Their purpose is to consume bandwidth over the first hop in an ON-OFF fashion. As in experiment 1 , they are assumed to generate ADUs of length equal to 1 cell with probability $p=0.5$ and equal to $L P_{\max }=20$ with probability $1-p=0.5$. When in the OFF state they allow the DC policy to build deadline credit. When in the ON state they create a bottleneck for the rest of the streams over the first hop. 


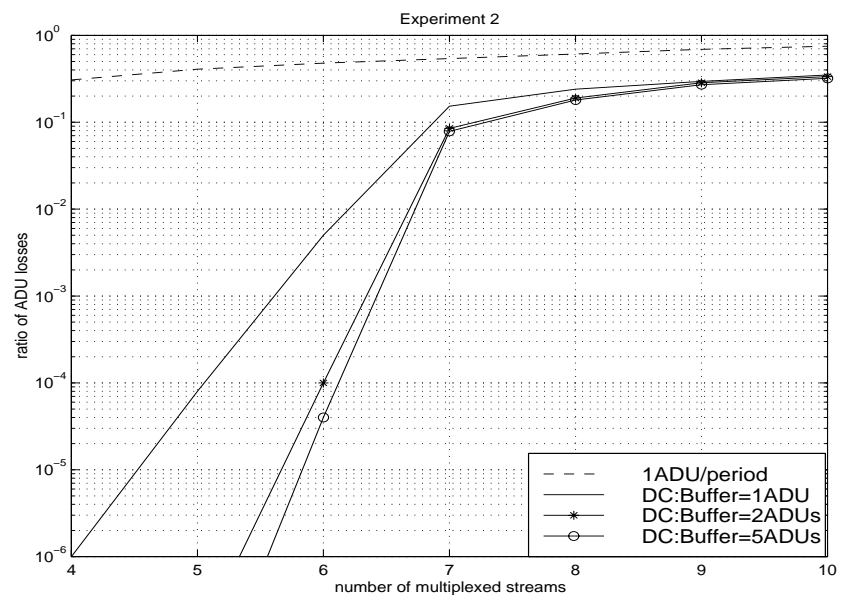

Fig. 3. Experiment 2: Ratio of induced ADU losses vs number of multiplexed streams (not including background traffic).

The objective of this scenario is to show that the DC algorithm dynamically builds deadline credit by utilising the available bandwidth and buffer space at nodes 2 and 3 .

Fig. 3 shows the ratio of the induced ADU losses versus the number of multiplexed streams (not including the background traffic streams). Over the first hop (node 1 to node 2) the total number of streams is always the number of streams indicated in the $\mathrm{x}$-axis plus three background traffic streams. Over the second hop (node 2 to node 3 ) the total number of streams is the number indicated on the x-axis. As in Experiment 1, there is a significant improvement in the induced ADU losses between the two approach es and for the case of the DC policy, the performance improves with increasing available buffer space.

\section{References}

1. Klara Nahrstedt Ian R. Philp and Jane W.S. Liu. Scheduling and Buffer Management for Soft-Real-Time VBR Traffic in Packet-Switched Networks. Technical Report, University of Illinois at Urbana-Champaign, Ill, 1996.

2. George Kesidis. ATM Network Performance. Kluwer Academic Publisher, 1996.

3. J.M. Mc Manus and K.W. Ross. Video on Demand over ATM: ConstantRate Transmission and Transport. Technical Report, University of Pennsylvania, Philadelphia, PA, http://www. seas.upenn.edu/ ${ }^{\sim}$ ross/, November 1995.

4. Zoe Antoniou and Ioannis Stavrakakis. Earliest Due First Scheduling for Application-Level QoS Delivery. Proceedings of IEEE Conference on Protocols for Multimedia Systems - Multimedia Networking, pp.172-182, Santiago, Chile, November 1997.

5. Sujata Banerjee. Translating Application Requirements to ATM Cell Level Requirements. Proceedings of IEEE International Conference on Communications, 1997. 
6. Z. Antoniou and I. Stavrakakis. Deadline Credit Scheduling Policy for Pre-recorded Sources. To appear in Proceedings of GLOBECOM '99, December 5-9 1999.

7. Z. Antoniou and I. Stavrakakis. Efficient End-to-End Transport of Soft Real Time Applications. Report, CDSP Centre, Northeastern University, 1999.

8. J.M. Mc Manus and K.W. Ross. Prerecorded VBR Sources in ATM Networks: Piecewise-Constant-Rate Transmission and Transport. Technical Report, University of Pennsylvania, Philadelphia, PA, http://www.seas.upenn.edu/ ross/, September 1995. 